\title{
Avaliação do perfil químico e biológico de frutos de Hylocereus polyrhizus
}

\author{
Evaluation of the chemical and biological profile of Hylocereus polyrhizus fruits \\ Evaluación del perfil químico y biológico de frutos de Hylocereus polyrhizus
}

Recebido: 15/07/2021 | Revisado: 20/07/2021 | Aceito: 22/07/2021 | Publicado: 31/07/2021

\author{
Antônio Maia Rodrigues \\ ORCID: https://orcid.org/0000-0003-4726-8314 \\ Universidade Nilton Lins, Brasi \\ E-mail: toinhomrg884@gmail.com \\ Roosevelt Guimarães Soares Júnior \\ ORCID: https://orcid.org/0000-0002-1201-5547 \\ Universidade Nilton Lins, Brasil \\ E-mail: junioroosevelt@gmail.com \\ Gabriel Oliveira de Souza \\ ORCID: https://orcid.org/0000-0001-9168-074X \\ Universidade Nilton Lins, Brasil \\ E-mail: gabriel.souza@uniniltonlins.edu.br
}

\begin{abstract}
Resumo
O potencial farmacológico proveniente de bioativos naturais, tem impulsionado cada vez mais o mercado farmacêutico, que busca potencializar terapias e implementar novas moléculas e produtos bioativos. Nesse contexto os frutos de Hylocereus polyrhizus tem apresentado um grande valor nutricional e um indicativo da presença de possíveis metabolitos secundários em sua composição. Diante deste contexto esta pesquisa visou o objetivo de avaliar o perfil químico e biológico de frutos de Hylocereus polyrhizus. As amostras foram adquiridas em supermercado da rede provada de Manaus- Am. Posteriormente foram estabilizadas e foram realizadas as análises propostas. Os resultados da analise químicas apontam a presença de compostos fenólicos como flavonoides, taninos, catequinas, flavonas, flavonóis e outros compostos fenólico com potencial antioxidante e consequentemente com potencial biológico para outras patologias relacionadas o estresse oxidativo. O teor de compostos fenólicos e flavonoides, apontam a presença dessas substancias, o que comprovam o resultado positivo da analise fitoquímica. Por fim a análise biológica frente aos radicais DPPH e ABTS apontam que os frutos de Hylocereus polyrhizus tem considerável ação antioxidante com base no IC50 apresentando principalmente frente ao radical ABTS, o que corrobora os resultados anteriores uma vez que compostos hidrofílicos, como fenóis e flavonoides, tendem a ter mais afinidade por esse radical. Podemos concluir que os frutos de Hylocereus polyrhizus tem um grande potencial farmacêutico, devido a presença de substancias antioxidantes que podem combater os radicais livres e diminuir o estresse oxidativo, que estão relacionadas a diversas patologias como diabetes, câncer e doenças degenerativas. Sem dúvida os frutos de Hylocereus polyrhizus tem são excelentes aliados terapêuticos dentro do contexto da fitoterapia.
\end{abstract}

Palavras-chaves: Frutos; Antioxidantes; Metabolitos secundários.

\begin{abstract}
The pharmacological potential from natural bioactives has increasingly driven the pharmaceutical market, which seeks to enhance therapies and implement new molecules and bioactive products. In this context, the fruits of Hylocereus polyrhizus have shown a great nutritional value and an indication of the presence of possible secondary metabolites in its composition. In this context, this research aimed to evaluate the chemical and biological profile of Hylocereus polyrhizus fruits. The samples were acquired in a supermarket of the private network of Manaus-Am. Later they were stabilized and the proposed analyzes were carried out. The results of the chemical analysis point to the presence of phenolic compounds such as flavonoids, tannins, catechins, flavones, flavonols and other phenolic compounds with antioxidant potential and consequently with biological potential for other pathologies related to oxidative stress. The content of phenolic compounds and flavonoids indicate the presence of these substances, which proves the positive result of the phytochemical analysis. Finally, the biological analysis against the DPPH and ABTS radicals indicate that the fruits of Hylocereus polyrhizus have considerable antioxidant action based on the IC50 presenting mainly against the ABTS radical, which corroborates the previous results since hydrophilic compounds such as phenols and flavonoids, tend to have more affinity for this radical. We can conclude that the fruits of Hylocereus polyrhizus have a great pharmaceutical potential, due to the presence of antioxidant substances that can fight free radicals and reduce oxidative stress, which are related to several pathologies such as diabetes, cancer and degenerative diseases. Undoubtedly the fruits of Hylocereus polyrhizus are excellent therapeutic allies within the context of phytotherapy.
\end{abstract}

Keywords: Fruits; Antioxidants; Secondary metabolites. 


\begin{abstract}
Resumen
El potencial farmacológico derivado de los bioactivos naturales ha impulsado cada vez más el mercado farmacéutico, que busca potenciar las terapias e implementar nuevas moléculas y productos bioactivos. En este contexto, los frutos de Hylocereus polyrhizus han mostrado un gran valor nutricional y un indicio de la presencia de posibles metabolitos secundarios en su composición. En este contexto, esta investigación tuvo como objetivo evaluar el perfil químico y biológico de frutos de Hylocereus polyrhizus. Las muestras se adquirieron en un supermercado de la red privada de Manaus-Am. Posteriormente se estabilizaron y se realizaron los análisis propuestos. Los resultados del análisis químico indican la presencia de compuestos fenólicos como flavonoides, taninos, catequinas, flavonas, flavonoles y otros compuestos fenólicos con potencial antioxidante y consecuentemente con potencial biológico para otras patologías relacionadas con el estrés oxidativo. El contenido de compuestos fenólicos y flavonoides indica la presencia de estas sustancias, lo que prueba el resultado positivo del análisis fitoquímico. Finalmente, el análisis biológico contra los radicales DPPH y ABTS indica que los frutos de Hylocereus polyrhizus tienen una acción antioxidante considerable basada en el IC50 presentándose principalmente contra el radical ABTS, lo que corrobora los resultados anteriores ya que los compuestos hidrófilos como fenoles y flavonoides, tienden a tener más afinidad por este radical. Podemos concluir que los frutos de Hylocereus polyrhizus tienen un gran potencial farmacéutico, debido a la presencia de sustancias antioxidantes que pueden combatir los radicales libres y reducir el estrés oxidativo, los cuales están relacionados con diversas patologías como diabetes, cáncer y enfermedades degenerativas. Sin duda, los frutos de Hylocereus polyrhizus son excelentes aliados terapéuticos en el contexto de la fitoterapia.
\end{abstract}

Palabras clave: Frutas; Antioxidantes; Metabolitos secundarios.

\title{
1. Introdução
}

Plantas são fontes de estruturas complexas, diversas e biologicamente ativas, resultantes do seu metabolismo secundário, as quais podem ser empregas na sua estrutura original ou quimicamente modificada na produção de medicamentos (Atasanov et al., 2015). De 1981 a 2014, 50\% das drogas aprovadas foram produtos naturais inalterados, derivados ou mimetizados, e fitoterápicos (Newman, 2016).

Foram descritas na espécie Hylocereus polyrhizus a presença de flavonoides, compostos naturais com alto poder antioxidante, sendo bastante utilizado para a produção de cremes faciais (Sarmento, 2017). Além de pectinas (Chua et al., 2018), alguns oligossacarídeos, que podem funcionar como probióticos naturais (Zhang et al., 2018) e algumas betacianinas com potencial antioxidante, e relacionado ao combate do diabetes (Song et al., 2015).

O gênero Hylocereus, são frutas originárias do México e cultivadas, principalmente em países tropicais ou subtropicais, como Taiwan, Sul da China, Israel, Tailândia Austrália e América Latina. Atualmente, são consideradas frutas exóticas, tendo em vista a sua casca contendo aspecto escamoso e a polpa com intensa coloração rosada, utilizada também como corante natural, ganhando o nome de fruta-dragão (Verona-Ruiz, 2020).

Com base nas propriedades farmacológicas descritas acerca do uso de frutos de Hylocereus polyrhizus, esta pesquisa teve como objetivo avaliar o perfil químico e biológico de frutos de Hylocereus polyrhizus.

\section{Metodologia}

\subsection{Amostragem}

A natureza da pesquisa foi aplicada com abordagem mista, pois apresentou características qualitativas e quantitativas (Gehardt \& Silveira, 2009). O material fresco de Hylocereus polyrhizus foi adquirido em rede de supermercado privado na cidade de Manaus, Amazonas, posteriormente foi higienizado com a submersão da amostra em $4 \mathrm{~L}$ de água destilada e $60 \mathrm{ml} \mathrm{de}$ hipoclorito de sódio, onde ficou na solução por 15 minutos e em seguida lavado novamente com água corrente. Após a higienização, o produto foi cortado em pequenos cubos e colocado à $60^{\circ} \mathrm{C}$ em estufa de circulação de ar para secagem por 96 horas (Ministério da Saúde, 2008). 


\subsection{Avaliação do Perfil Químico de Frutos de Hylocereus polyrhizus}

\subsubsection{Avaliação Fitoquímica}

O extrato hidroetanólico foi submetido aos testes fitoquímicos descritos por Matos (2009) de reações colorimétricas e físico-químicas, no entanto foram realizadas algumas adaptações.

\subsubsection{Prospecção de Antraquinonas}

Foram postas $0,5 \mathrm{~g}$ da amostra vegetal em um tubo de ensaio e adicionado $10 \mathrm{~mL}$ de etanol 70\%, e logo após foi adicionado mais $1 \mathrm{~mL}$ de $\mathrm{NH}_{4} \mathrm{OH} 10 \%$ e realizou-se a agitação da solução. Observou-se se ouve o aparecimento de cor rosa ou vermelho-cereja (indicativo de antraquinonas).

\subsubsection{Identificação de Cumarinas}

Despejou-se, separadamente, duas gotas do extrato recém preparado sobre um pedaço de papel filtro comum, uma gota para mancha teste e outra gota para mancha controle. Em seguida, sobre a mancha teste, foi gotejado uma solução de hidróxido de potássio $10 \%$ em etanol $1 \mathrm{~N}$ e sob luz UV $365 \mathrm{~nm}$, foi observado se ouve o aparecimento de fluorescência amarelo-esverdeada na mancha teste (indicativo positivo de cumarinas).

\subsubsection{Identificação de Taninos}

Foram adicionados $30 \mathrm{~mL}$ do extrato etanólico $70 \%$ em um béquer que foi submetido ao banho maria até que ocorresse a evaporação do álcool, em seguida foi filtrado e deixou-se arrefecer. Foi transferido o restante do extrato aquoso para um balão volumétrico de $25 \mathrm{~mL}$ e avolumou-se. Logo após foram adicionadas 2 gotas de solução aquosa de cloreto férrico $2 \%$ ao tubo, a solução foi agitada e observou-se a formação de precipitados, sendo precipitado azul para taninos hidrolisáveis e precipitado verde para taninos condensados.

\subsubsection{Identificação de Antocianinas, Antocianidinas, Flavonas, Flavonóis, Xantonas, Chalconas, Auronas e Flanonois}

Foi realizado o ajuste do $\mathrm{pH}$ dos tubos 2, 3 e 4 para 3, 8,5 e 11 por meio de ácido sulfúrico 2 Molar, hidróxido de sódio $2 \mathrm{~N}$ e hidróxido de potássio $2 \mathrm{~N}$, respectivamente.

Logo após observou-se e interpretou-se o aparecimento de cores conforme o Quadro 1.

Quadro 1: Reação colorimétrica para compostos flavônicos variados.

\begin{tabular}{cccc}
\hline CONSTITUINTES & TUBO 2 & TUBO 3 & TUBO 4 \\
\hline Antocianinas e Antocianidinas & Vermelho & Lilás & Azul-purpura \\
\hline Flavonas, Flavonois e Xantonas & - & - & Amarelo \\
\hline Chalcona e Auronas & Vermelho & - & Vermelho-purpura \\
\hline Flavonois & - & - & De vermelho a laranja \\
\hline
\end{tabular}

Fonte: Autores.

\subsubsection{Teste para Leucoantocianinas, Catequinas e Flavanonas}

Foi realizado o ajuste do $\mathrm{pH}$ dos tubos 5 e 6 a 1 e 11 por meio da adição de ácido clorídrico $2 \mathrm{~N}$ e hidróxido de sódio $2 \mathrm{~N}$, respectivamente. Logo após observou-se e interpretou-se o aparecimento de cores conforme a Quadro 2. 
Quadro 2: Reação colorimétrica para leucoantocianinas, catequinas e flavanonas.

\begin{tabular}{ccc}
\hline CONSTITUINTES & TUBO 5 & TUBO 6 \\
\hline Leucoantocianinas & Vermelho & - \\
\hline Catequinas (taninos catéticos) & Pardo-Amarelado & - \\
\hline Flavonas & - & De vermelho a laranja \\
\hline
\end{tabular}

Fonte: Autores.

\subsubsection{Prospecção Fitoquímica para Triterpenos, Esteroides e Cardioativos}

Foram adicionados $50 \mathrm{~mL}$ do extrato etanólico $70 \%$ em um béquer com mais $20 \mathrm{~mL}$ de acetato de chumbo a $10 \%$, e realizou-se a decocção por 3 minutos. Foi extraído com três porções de $10 \mathrm{~mL}$ de clorofórmio, reunindo as fases orgânicas. Distribuíram-se alíquotas de $5 \mathrm{~mL}$ em 6 tubos de ensaio e submeteu-se os tubos aos testes abaixo.

\subsection{Identificação do Núcleo Lipídico}

\subsection{Reação de Libermann-Burchard (Esteroides e Triterpenos)}

Foi adicionado $1 \mathrm{~mL}$ de anidrido acético mais 3 gotas de ácido sulfúrico e agitou-se suavemente. Logo após foi observado se ocorreu o aparecimento das cores vermelha, rosa, púrpura ou violeta para núcleo triterpênico, e azul ou verde para núcleo esterioidal.

\subsection{Identificação de Glicosídeos Cardioativos}

Esse teste foi realizado somente nos tubos com resultados positivos para núcleos esterioidais.

\subsection{Identificação da y-lactona}

\subsection{Reação de Kedde}

Foram adicionados $2 \mathrm{~mL}$ reativo de Kedde e 2 gotas de hidróxido de potássio em etanol e aguardaram-se 5 minutos para que fosse observado o desenvolvimento de coloração vermelho-violeta estável.

\subsection{Reação de Baljet}

Foram adicionados $2 \mathrm{~mL}$ reativo de Baljet e 2 gotas de hidróxido de potássio em etanol e observou-se o aparecimento da cor laranja estável.

\subsubsection{Prospecção das Saponinas}

Preparou-se $100 \mathrm{~mL}$ de extrato aquoso a $2 \%$ de droga vegetal por infusão ou decocção e deixou-se arrefecer. Transferiram-se $5 \mathrm{~mL}$ para um tubo de ensaio, logo após foi realizada agitação por 15 segundos para que fosse observado a formação de espuma persistente por 15 minutos. Em seguida foram adicionadas gotas de ácido clorídrico $2 \mathrm{~N}$ sobre a espuma e observou-se se a espuma continuava persistente.

\subsubsection{Prospecção Fitoquímica para Compostos Nitrogenados}

\subsection{Extração}

Preparou-se um extrato por decocção a partir de $1 \mathrm{~g}$ da matéria prima vegetal e $20 \mathrm{~mL}$ de ácido clorídrico a $10 \%$. 
$\mathrm{O}$ extrato foi filtrado em um funil de separação e alcalinizado até pH 11 com hidróxido de amônio. Em seguida foi adicionado $10 \mathrm{~mL}$ de éter etílico e agitado. Deixou-se repousar até que ocorresse a separação das fases. Retirou-se a fase orgânica e realizou-se a evaporação da mesma para que fosse ressuspendido com $20 \mathrm{~mL}$ de ácido diluído.

\subsubsection{Teste para Alcaloide}

Distribuíram-se $2 \mathrm{~mL}$ da fase aquosa acidificada em 4 tubos de ensaio e acrescentaram-se 3 gotas dos reagentes gerais a cada tubo, observando a formação de precipitados.

\subsection{Doseamento de Fenóis Totais}

Os extratos foram analisados pelo método de Folin-Ciocalteu com pequenas modificações. Uma alíquota de $10 \mu \mathrm{L}$ de cada uma das soluções das amostras e do padrão epicatequina a $1 \mathrm{mg} / \mathrm{mL}$ em etanol foi transferida em triplicata para uma placa de 96 poços. Em seguida, foram adicionados $50 \mu \mathrm{L}$ solução de Folin-Ciocalteau (1:10 em água destilada), e então a placa foi incubada em temperatura ambiente por 8 minutos. Ao final, foram adicionados $240 \mu \mathrm{L}$ de carbonato de sódio $0,4 \%$ e a placa foi novamente incubada por mais 3 minutos. A absorbância foi medida a $620 \mathrm{~nm}$ usando um espectrofotômetro. A quantidade de fenóis foi expressa em equivalente de epicatequina por grama de amostra (Guilhon-Simplicio et al., 2011).

\subsection{Doseamento de Flavonoides Totais}

Inicialmente, os extratos foram diluídos a $1 \mathrm{mg} / \mathrm{mL}$ em etanol, então $30 \mu \mathrm{L}$ dessas soluções foram transferidas para uma placa de 96 poços, em triplicata. À mesma placa foram transferidos $30 \mu \mathrm{L}$ de uma solução de quercetina a $1 \mathrm{mg} / \mathrm{mL} \mathrm{em}$ etanol 80\%, padrão da análise. Depois disso foram adicionados $90 \mu \mathrm{L}$ de etanol $95 \%, 6 \mu \mathrm{L}$ de cloreto de alumínio a $10 \%, 6$ $\mu \mathrm{L}$ de acetato de potássio $1 \mathrm{M}$ e $168 \mu \mathrm{L}$ de água destilada em cada poço. A placa foi incubada por 30 minutos em temperatura ambiente e a absorbância medida a $405 \mathrm{~nm}$ em um espectrofotômetro. A quantidade de flavonoides foi expressa em equivalente de quercetina por grama de amostra (Guilhon-Simplicio et al., 2011).

\subsection{Avaliação do Potencial Biológico}

\subsubsection{Ensaio de DPPH}

Inicialmente, a absorbância da solução de DPPH foi ajustada para 1,0 por meio de diluições com etanol a $50 \%$ seguidas de medidas de absorbância no comprimento de onda de $517 \mathrm{~nm}$, o mesmo para todo o teste. Foram distribuídas alíquotas de 250,0 $\mu \mathrm{L}$ das diluições dos extratos em triplicata em microplaca, e as absorbâncias foram medidas. Essa leitura constituiu o branco nos cálculos. Em seguida, foram acrescidos 100,0 $\mu \mathrm{L}$ da solução de DPPH em todos os poços com extrato. Após 30 minutos de incubação no escuro e à temperatura ambiente, as absorbâncias foram novamente medidas. O controle negativo foi constituído de $250,0 \mu \mathrm{L}$ de etanol a 50\% e 100,0 $\mu \mathrm{L}$ da solução de DPPH, em triplicata, também incubado nas mesmas condições. A atividade antioxidante, apresentada como concentração inibitória a 50 \% ( $\left.\mathrm{CI}_{50}\right)$, foi calculada em relação ao controle negativo com base na formula 1 (Guilhon-Simplicio et al., 2011).

Fórmula 1: Formula para o cálculo da capacidade antioxidante do extrato.

$$
\% \text { Inibição }=100[(\text { Abs2 amostra }- \text { Abs1 amostra }) / \text { Abs controle }]
$$

Fonte: Autores (2021) 


\subsubsection{Ensaio do Radical Livre ABTS}

Primeiramente, foi realizado uma diluição a $40 \%$ da solução de ABTS recém-preparada, com água mili-Q. Alíquotas de 250,0 $\mu \mathrm{L}$ das diluições dos extratos foram distribuídas em triplicata em microplaca e as absorbâncias foram medidas no comprimento de onda de $734 \mathrm{~nm}$. Essa leitura constituiu o branco nos cálculos. Em seguida, foram acrescidos 100,0 $\mu \mathrm{L}$ da solução de ABTS em todos os poços com extrato. Após 15 minutos de incubação no escuro e à temperatura ambiente, as absorbâncias foram novamente medidas. O controle negativo foi constituído de $250,0 \mu \mathrm{L}$ de etanol a $50 \%$ e $100,0 \mu \mathrm{L}$ da solução de ABTS, também incubado nas mesmas condições. A atividade antioxidante foi calculada em relação ao controle negativo com uso da formula 2 (Guilhon-Simplicio et al., 2011).

Fórmula 2: Formula para o cálculo da capacidade antioxidante do extrato

$$
\% \text { Inibição }=100[(\text { Abs2 amostra }- \text { Abs1 amostra }) / \text { Abs controle }]
$$

Fonte: Autores (2021).

\section{Resultados e Discussão}

$\mathrm{Na}$ análise do perfil químico pela triagem fitoquímica de metabolitos secundários dos frutos de H. polyrhizus (tabela 1), pode-se verificar que muitas classes de compostos estão presentes no extrato pesquisado, tais como compostos fenólicos, taninos condensados, flavonóis, flavonas e as catequinas que reafirmam a presenta de taninos condensados uma vez que as mesmas são precursoras dos mesmos, por reação de conjugação química na via do ácido chiquímico.

Tabela 1: Resultados da avaliação fitoquímica de frutos de H. polyrhizus.

\begin{tabular}{c|c}
\hline Metabólito Secundário & Resultado \\
\hline Compostos Fenólicos & Presente \\
\hline Antraquinonas & Ausente \\
\hline Cumarinas & Ausente \\
\hline Taninos Hidrolisáveis & Ausente \\
\hline Taninos Condensados & Presente \\
\hline Antocianinas e Antocianidinas & Ausente \\
\hline Flavonas, Flavanóis e Xantonas & Presente \\
\hline Chalcona e Auronas & Ausente \\
\hline Flavonois & Presente \\
\hline Leucoantocianidinas & Ausente \\
\hline Catequinas & Presente \\
\hline Flavanonas & Presente \\
\hline Esteroides & Presente \\
\hline Triterpenos & Ausente \\
\hline Slicosídeos Cardioativos & Ausente \\
\hline Alcaloides & Ausente \\
\hline
\end{tabular}

Fonte: Autores (2021). 
A presença de taninos condensados na amostra foi confirmada através da formação de precipitados verdes (Matos, 2009). Eles são classificados em dois grupos de acordo com a sua estrutura química, são esses: taninos hidrolisáveis e taninos condensados. Ambos os tipos são encontrados em quase todas as partes das plantas: raízes, rizomas, lenho, cascas, sementes, frutos e folhas. A complexação entre taninos e proteínas é a base para suas propriedades como fatores de controle de insetos, fungos e bactérias tanto quanto para suas atividades farmacológicas. Os taninos, assim como outras substâncias fenólicas, possuem atividade antioxidante, muito usadas na cosmetologia, pelo seu perfil químico. Plantas ricas em taninos são empregadas na medicina tradicional para o tratamento de diversas enfermidades, como diarreias, pressão alta, reumatismo, hemorragias, feridas, queimaduras, problemas estomacais (azia, náusea, gastrite e úlcera gástrica), problemas renais e do sistema urinário e processos inflamatórios em geral (Simões, 2007).

As reações para a pesquisa de esteroides se mostraram positivas com o aparecimento da cor azul, exatamente como descreve a metodologia de Matos (2009). Dentre os benefícios à saúde humana destacam-se a diminuição nos níveis de colesterol no sangue; redução nos riscos de desenvolvimento de doenças cardiovasculares e inibição do surgimento de certos tipos de tumores malignos (Pereira; Cardoso, 2012 apud Cunha, 2016).

$\mathrm{O}$ extrato ainda apresentou de acordo com a triagem fitoquímica realizada a presença de diversos compostos fenólicos tais como flavonóis, flavonas, catequinas, Flavonas e Xantonas. As propriedades terapêuticas dos flavonoides associadas à sua ação como antioxidante têm sido extensivamente estudadas. Sabe-se que é uma dieta rica em antioxidantes previne doenças associadas ao stress oxidativo e o envelhecimento (Degáspari \& Waszczynsky, 2004) e a incidência de alguns tipos de câncer (Sandhar et al., 2011).

A literatura descreve bem atividade antioxidante e anti-inflamatória dos flavonoides em relação sua estrutura (Figura 1). As principais características químicas e estruturais que conferem aos flavonoides sua importante atividade antioxidante são:

- Substituintes doadores de hidrogênio (grupos hidroxílicos fenólicos), que permitem aos flavonoides suprir uma reação de redução que ajuda a inibir a formação de radicais livres, isso mais facilmente na posição C3 e C5 (figura). (Sroka et al., 2015)

- Um sistema de deslocamento estável (de duplas conjugadas) que ajudam a deslocar a distribuição eletrônica na molécula. Principalmente uma instauração em C2 e C3 em conjunção com a função 4-oxo do anel C (figura) (Pisonero-Vaqueroi et al., 2014).

Figura 1: Principais características estruturais requeridas para a atividade antioxidante das moléculas de flavonoides.

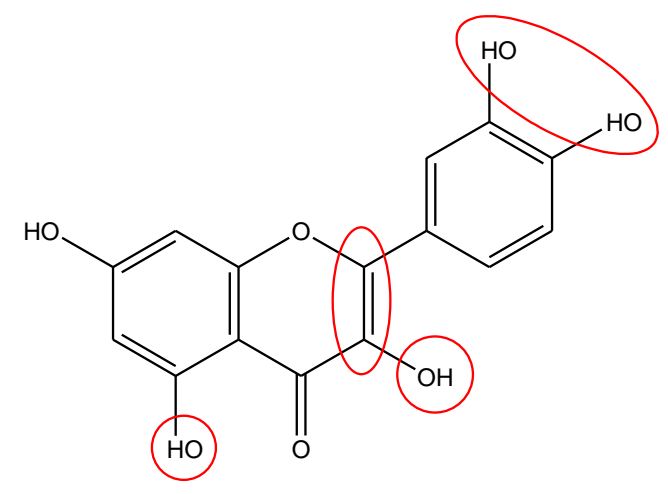

Fonte: Autores, elaborado no software chemdraw 18.0 (2021).

Diante do perfil de compostos fenólicos apresentados pelo extrato, seguiu-se os testes com a determinação do teor de compostos fenólicos bem como o perfil antioxidante do extrato. 


\subsection{Teor de Fenóis e Flavonoides Totais}

Foram pesquisados no extrato o teor de fenólicos e flavonoides afim de explorar a ação antioxidante presente nesses compostos. A amostra de H. polyrhizus submetida aos testes evidenciou uma alta concentração, como pode ser visto na Tabela 2:

Tabela 2: Teor de fenóis e flavonoides nas amostras de $H$. polyrhizus.

\begin{tabular}{cccc}
\hline Espécie & Extrato & Fenóis $(\boldsymbol{\mu g} \mathbf{E E} * \mathbf{m l})$ & $\begin{array}{c}\text { Flavonoides }(\boldsymbol{\mu g} \\
\mathbf{E Q *} / \mathbf{m l})\end{array}$ \\
\hline H. polyrhizus & Hidroetanólico & $248,20 \pm 0,89$ & $7,87 \pm 0,64$ \\
\hline
\end{tabular}

*EE: Equivalentes de epicatequina **EQ: Equivalentes de quercetina, Fonte: Autores (2021).

Os resultados encontrados evidenciam a presença de flavonoides e compostos fenólicos. Comparando o valor de

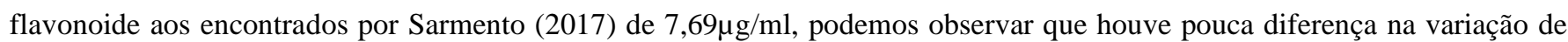
concentração, o que pode ser explicado pelo meio em que a espécie foi cultiva, visto que no estudo em questão, as amostras foram colhidas de forma fresca no semiárido, enquanto as amostras desse trabalho foram coletadas em comércio. Além disso, esse fator pode ser explicado pelos fatores intrínsecos e extrínsecos que atuam na variabilidade química dos metabólitos secundários em plantas, tais como: disponibilidade de água, sazonalidade e tipo genético da planta. O processo de estabilização e melhor metodologia de extração também atuam como determinantes na eficiência dos processos extrativos e teor desses metabolitos (Abílio 2011).

O maior destaque deve ser que esses valores de teores elevados de compostos fenólicos e flavonoides, confirmam os resultados encontrados nos testes de triagem fitoquímica (Marques et al, 2011).

Quanto à atividade antioxidante, realizada pelo método 2,2 difenil-1-picril hidrazil radical (DPPH) e do radical livre ABTS, os resultados se encontram na Tabela 3:

Tabela 3: Valores de DPPH e ABTS da amostra de H. polyrhizus comparadas ao padrão quercetina.

\begin{tabular}{llll}
\hline \multirow{2}{*}{ Amostra } & Extrato & DPPH & ABTS \\
\cline { 3 - 4 } & & CI50 em $\mu \pm \sigma \mu \mathrm{g} / \mathrm{ml}$ & \\
\hline H. polyrhizus & Hidroetanólico & $3,00 \pm 0,58$ & $0,51 \pm 0,00$ \\
\hline Quercetina & - & $4,10 \pm 0,80$ & $2,8 \pm 0,4$ \\
\hline
\end{tabular}

Fonte: Autores (2021).

Os resultados foram expressos utilizando o valor de concentração efetiva (CI50), que representa a concentração de amostra necessária para neutralizar 50\% dos radicais de DPPH e ABTS. Sendo assim quanto menor o CI50, maior a eficácia antioxidante da amostra (Guilhon-Simplicio et al., 2011).

O padrão utilizado para a mensuração dos resultados foi a quercetina $(3,5,7,3$ ' - 4 ' - pentahidroxi flavona), uma subclasse dos flavonoides que apresenta um alto teor de atividade antioxidante agindo sobre a ação da contra-peroxidação lipídica, interrompendo a reação em cadeia de formação de radicais livres (Marques et al., 2011).

A amostra de $H$. polyrhizus apresentou um baixo valor de CI50 quando comparada com o padrão quercetina, isso porque, provavelmente, haja a presença de mais de um tipo de flavonoide, que ocasionaria no fenômeno de sinergia, onde várias moléculas que exercem a mesma função irão potencializar a sua execução (Abílio, 2011).

Pela primeira vez na literatura foram realizados ensaios com o radical ABTS, onde mostram resultados de atividade 
antioxidante superiores aos encontrados com o radical DPPH (tabela 3). Uma vez que quantidade ainda mais menores foram necessárias para se inibir 50\% do radical de acordo com IC50 calculado.

Ensaios químicos de atividade antioxidante são úteis para avaliação inicial do potencial de neutralização de radicais livres de diversas sustâncias. Nesses ensaios é possível avaliar se as substâncias atuam por transferência de átomos de hidrogênio ou de elétrons. Nesse sentido, ensaios como o do $\mathrm{DPPH}^{\bullet}$ (que avalia a capacidade de doação de átomos de hidrogênio), $\operatorname{ABTS}^{\bullet+}$ (que avalia a capacidade de doação de elétrons e hidrogênio) são populares pela simplicidade, rapidez, reprodutibilidade e baixo custo (Gülçin, 2012).

Os radicais livres estão associados a diversos processos degenerativos e patogênicos como o envelhecimento, doenças coronarianas, câncer (Degáspari \& Waszczynsky, 2004) artrites inflamatórias, úlceras, diabetes, doença de Alzheimer, doença de Parkinson, aterosclerose, entre outros. (Aquino, 2017).

De forma geral, as baixas $\mathrm{CI}_{50}$ das nossas amostras nos ensaios do DPPH e ABTS, sugerem grande potencial antioxidante destas espécies. Adicionalmente, indica que estas amostras podem atuar doando elétrons ou hidrogênios, o que é compatível com o mecanismo de ação da atividade antioxidante de compostos fenólicos (Leopoldini et al., 2011).

\section{Conclusão}

A biodiversidade amazônica confere ao Brasil uma posição estratégica na extração e produção novos bioativos, pelas diversas propriedades terapêuticas de extratos presentes nas plantas amazônicas. Assim, com a evolução e crescimento do mercado nacional e internacional, a pesquisa e desenvolvimento de biocompostos torna-se primordial para a ampliação desse setor.

A extração hidroetanólica dos frutos de H. polyrhizus apresentou em sua composição importantes metabolitos como os fenóis, taninos, flavonoides, esteroides, flavonois, flavanonas e catequinas, o que demonstra que a amostra testada pode possuir um grande interesse farmacêutico no ramo da fitoterapia ou da farmacologia.

A ação antioxidante da amostra se mostrou elevada comparada com o padrão quercetina utilizado, evidenciando a alta eficácia farmacológica que esse produto pode exercer sobre o organismo. Por tanto se faz necessário mais estudos que aprofundem o estudo dessa ação antioxidante, principalmente frente ao radical ABTS.

Os frutos de $H$. polyrhizus demonstram um proeminente potencial químico para futuros estudos de atividades enzimáticas nos campos farmacológico e nutricionais dado seu perfil de metabolitos, além de possíveis contribuições no campo da fitoterapia devido seu provável sinergismo químico demonstrado nas atividades antioxidantes preliminares.

\section{Referências}

Abílio, G. M. F. (2011). Plantas Medicinais. Bananeiras: Editora Universitária/UFPB, 6.

Aquino, et al. (2017). Metabólitos e ação antioxidante de Croton Heliotropiifolius e Croton blanchetianus. Acta Brasiliensis 1(3), 7-10.

Atanasov, A. G. et al. (2015). Discovery and resupply of pharmacologically active plant-derived natural products: A review. Biotechnol. Advance. 33(8), $1582-1614$

Chua, B., Ng, Y. \& Ali, A. (2018). Ultrasound Assisted Extraction of Pectin from Dragon Fruit Peels. Journal of Engineering Science and Technology. 65-81.

Degáspari, C. H. \& Waszczynsky, J. N. (2004). Propriedades antioxidantes de compostos fenólicos. Visão Acadêmica. 5.

Gerhardt, T. E. \& Silveira, D. T. (2009) Métodos de Pesquisa. Editora da UFRGS. 120 p.

Gülçin, I. (2012). Antioxidant activity of food constituents: an overview. Arch Toxicol. 86(3), 345-91.

Leopoldini M., N. Russo M. Toscano. (2011). The molecular basis of working mechanism of natural polyphenolic antioxidants. Food Chemistry, 125:288-300.

Marques, V. B., Moreira, R. A., Ramos, J. D., Araújo, N. A. \& Silva, F. O. R. (2011). Fenologia reprodutiva de pitaia-vermelha no município de Lavras-MG. Ciência Rural, 41(6), 984-987. 
Research, Society and Development, v. 10, n. 9, e47110918290, 2021

(CC BY 4.0) | ISSN 2525-3409 | DOI: http://dx.doi.org/10.33448/rsd-v10i9.18290

Matos, F. J. A. (2009). Introdução a fotoquímica experimental. (3a ed.).

Ministério da Saúde. Guia Alimentar Para a População Brasileira: promovendo a alimentação saudável.

Newman, D. J. \& Cragg, G. M. (2016). Natural Products as Sources of New Drugs from 1981 to 2014. J. Nat. Prod. 79: 629-661.

Pereira, R. J. \& Cardoso, M. G. (2017). Metabólitos secundários vegetais e benefícios antioxidantes. J. Biotec. Biodivers. 3(4), 146-152.

Pisonero-Vaquero, S., Garcia-Mediavilla, M., Jorquera, F., Majano, P., Benet, M., Jover, R., Gonzalez-Galleno, J. \& Sanchez-Campos, S. (2014). Modulation of PI3K-LXR $\alpha$-dependent lipogenesis mediated by oxidative/nitrosative stress contributes to inhibition of HCV replication by quercetin. Lab. Invet. 94: 262274.

Santos, D. S. \& Rodrigues, M. M. F. Atividades farmacológicas dos flavonoides: um estudo de revisão. Estação Científica - UNIFAP. 7(3), 29-35.

Sarmento, J. D. A. (2017). Qualidade, Compostos Bioativos e Conservação Da Pitaia (Hylocereus Polyrhizus) No Semiárido Brasileiro. Universidade Federal Rural do Semi-Árido, Mossoró. 146p.

Sandhar, H. K., Kumar, B., Prasher, S., Tiwari, P., Salhan, M. \& Sharma, P. (2011). A Review of Phytochemistry and Pharmacology of Flavonoids. Internationale Pharmaceutica Sciencia, 1(1), $25-41$.

Simões, C. M. O. et al. (2007). Farmacognosia: da planta ao medicamento. (6a ed.), UFSC.

Simplício, F. G. \& Pereira, M. M. (2011). Aspectos Químicos e Farmacológicos de Byrsonima (Malpighiaceae). Quim Nova, 34(6), $1032-1041$.

Song, H., Chu, Q. \& Xu, D. et al. (2015). Purified Betacyanins from Hylocereus undatus Peel Ameliorate Obesity and Insulin Resistance in High-Fat-Diet-Fed Mice. Journal of Agricultural and Food Chemistry 64: 236-244.

Sroka, Z., Zbikowska, B. \& Hladyszowski, J. (2015). The antiradical activity of some selected flavones and flavonols. Experimental and quantum mechanical study. J. Mol. Model, 21:307.

Verona-Ruiz, A., Urcia-Cerna, J. \& Paucar-Menacho, L. Pitahaya (2020). (Hylocereus spp.): Cultivo, características fisicoquímicas, composición nutricional y compuestos bioactivos. Scientia Agropecuaria, 11(3), 439-453.

Zhang, S., Hu, H. \& Wang, L. et al. (2018). Preparation and prebiotic potential of pectin oligosaccharides obtained from citrus peel pectin. Food Chemistry 244: $232-237$. 